\title{
Respiratory Protection in Dentistry During the COVID-19 Outbreak
}

\section{Vanessa Paiva Reis ${ }^{1}$}

${ }^{1}$ Oral Maxillofacial Surgery and Traumatology Service, Military Police Polyclinic of Cascadura (Policlínica da Polícia Militar de Cascadura), Rio de Janeiro State Military Police, Rio de Janeiro, RJ, Brazil

- Conflicts of interest: none declared.

\section{Dear Editor,}

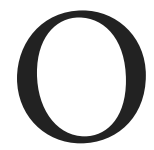
n January 8, 2020, the Chinese Center for Disease Control and Prevention officially announced a new coronavirus as the etiological agent of a new respiratory disease in humans. ${ }^{1}$ The disease rapidly spread around the world and on March 11, 2020, it was officially declared a SARS-CoV-2 pandemic by the World Health Organization.

The potential transmission of the virus via aerosols, droplets, and contact is crucially important for dental surgeons, since many of the procedures they perform generate aerosols. ${ }^{2,3}$ There is a consensus in the literature that N95/FFP2/FFP3 (FFP - Filtering Facepiece) and similar respirators offer better protection than disposable surgical masks because they filter more effectively and seal to the face (Chart 1). As such, they should be the option of choice for aerosol-generating procedures. ${ }^{4}$

However, there is a lack of consensus in the recommendations when it comes to respiratory protection against COVID-19 during non-aerosolgenerating procedures. An analysis of the public health recommendations issued in Brazil, the USA, the UK, Canada, and Australia, as well as the recommendations of the American Dental Association, revealed that some recommend N95/FFP2/FFP3 respirators for all dental procedures, ${ }^{4,5,6}$ while others recommend disposable surgical masks for non-aerosol-generating procedures. ${ }^{7,8,9,10,11}$
The respiratory protection recommendations found in the documents analyzed with the respective countries and dates of publication (latest update) are given in Table 1 .

Recent evidence indicates that SARS-CoV-2 may be transmitted via infectious aerosols produced naturally in speech and breathing (bioaerosols). ${ }^{12,13}$ While there is no scientific evidence that the viral load present in such bioaerosols is enough to cause infection, airborne transmission would explain the high contagion rates found in SARS-CoV-2. ${ }^{12}$ In view of the proximity of dental surgeons to the patient's face during dental treatments and their duration of exposure in an indoor environment, I question whether surgical masks provide effective enough filtering of potentially infectious particles in bioaerosols during dental treatments.

Dental surgeons are at high risk of infection from SARS-CoV-2 because of the high viral load present in the upper airways and their potential exposure to biological material during aerosol-generating procedures. The potential for transmission during the incubation period and by asymptomatic patients makes it important to adjust biosecurity protocols for all patients, irrespective of whether they have a confirmed or suspected infection. I believe respiratory protection recommendations should be reviewed to include the use of N95 respirators in all dental procedures, whether or not they are aerosol-generating.

Chart 1. General characteristics of FFP (Filtering Facepiece) masks*

\begin{tabular}{|l|l|c|c|c|}
\hline Class & $\begin{array}{c}\text { Filter penetration limit } \\
\text { (at 95 L/min air flow) }\end{array}$ & Inward leakage & Typical Elastic Band & \multicolumn{1}{c|}{ Indications } \\
\hline FFP1 & $\begin{array}{l}\text { Filters at least } 80 \% \text { of airborne } \\
\text { particles }\end{array}$ & $<22 \%$ & Yellow & $\begin{array}{l}\text { Dust and/or Mists (mechanically } \\
\text { generated aerosols) }\end{array}$ \\
\hline FFP2 & $\begin{array}{l}\text { Filters at least 94\% of airborne } \\
\text { particles }\end{array}$ & $<8 \%$ & Blue or White & $\begin{array}{l}\text { Fumes (thermally generated } \\
\text { aerosols) and/or Biological Agents }\end{array}$ \\
\hline FFP3 & $\begin{array}{l}\text { Filters at least 99\% of airborne } \\
\text { particles }\end{array}$ & $<2 \%$ & Red & $\begin{array}{l}\text { Particles highly toxic (TL }<0.05 \mathrm{mg} / \\
\mathrm{m}^{3} \text { ) and/or of unknown toxicity }\end{array}$ \\
\hline
\end{tabular}

*European Committee for Standardization (www.cen.eu). Abbreviations: FFP (Filtering Facepiece); TL (Tolerance Limit) 
Table 1. Official documents, country, date of latest update, and recommended respiratory protection.

\begin{tabular}{|c|c|c|}
\hline Agency - Date of Document & $\begin{array}{l}\text { Respiratory Protection: } \\
\text { Non-aerosol-generating Procedures }\end{array}$ & $\begin{array}{l}\text { Respiratory Protection: } \\
\text { Aerosol-generating Procedures }\end{array}$ \\
\hline $\begin{array}{l}\text { Agência Nacional de Vigilância Sanitária4 (Brazil) } \\
\text { May 8, } 2020\end{array}$ & N95 respirator & N95 respirator \\
\hline $\begin{array}{l}\text { American Dental Association7 (USA) } \\
\text { April 1, } 2020\end{array}$ & Surgical mask & N95 respirator \\
\hline $\begin{array}{l}\text { Centers for Disease Control and Prevention }{ }^{5} \text { (USA) } \\
\text { April 27, } 2020\end{array}$ & N95 respirator & N95 respirator \\
\hline $\begin{array}{l}\text { Communicable Diseases Network Australia } \\
\text { (Australia) } \\
\text { May 5, } 2020\end{array}$ & Surgical mask & N95 respirator \\
\hline $\begin{array}{l}\text { European Centers for Disease Control and } \\
\text { Prevention }^{6} \text { (Europe) } \\
\text { February } 28,2020\end{array}$ & FFP2/FFP3 respirator & FFP2/FFP3 respirator \\
\hline $\begin{array}{l}\text { National Health Service }{ }^{9} \text { (UK) } \\
\text { April 15, } 2020\end{array}$ & Surgical mask & N95 respirator \\
\hline $\begin{array}{l}\text { World Health Organization }{ }^{10} \text { (Switzerland) } \\
\text { April 6, } 2020\end{array}$ & Surgical mask & N95 respirator \\
\hline $\begin{array}{l}\text { Public Health Agency of Canada }{ }^{11} \text { (Canada) } \\
\text { April 30, } 2020\end{array}$ & Surgical mask & N95 respirator \\
\hline
\end{tabular}

Abbreviation: FFP (Filtering Facepiece)

\section{References}

1. Li Q, Guan X, Wu P, Wang X, Zhou L, Tong Y, et al. Early transmission dynamics in Wuhan, China, of novel coronavirus-infected pneumonia. $\mathrm{N}$ Engl J Med. 2020;382(13):1199-1207.

2. Peng X, Xu X, Li Y, Cheng L, Zhou X, Ren B. Transmission routes of 2019$\mathrm{nCoV}$ and controls in dental practice. Int J Oral Sci. 2020; 12(1):1-6.

3. Meng L, Hua F, Bian Z. Coronavirus disease 2019 (COVID-19): Emerging and future challenges for Dental and Oral Medicine. J Dent Res. In Press 2020.

4. ANVISA. NOTA TÉCNICA No 04/2020. Orientações para serviços de saúde: medidas de prevenção e controle que devem ser adotadas durante a assistência aos casos suspeitos ou confirmados de infecção pelo novo coronavírus (SARS-COV-2). (Atualizada em 8/05/2020). [acesso em 21 mai 2020]. Disponível em: http://portal.anvisa.gov.br/documents/33852/271858/ Nota+T\%C3\%A 9cnica+n+04-2020+GVIMS-GGTES-ANVISA/ab5986603de4-4f14-8e6f-b9341c196b28

5. CDC. Coronavirus Disease 2019 (COVID-19): Dental Settings Interim Infection Prevention and Control Guidance for Dental Settings During the COVID-19 Response. [acesso em 3 mai 2020]. Disponível em: https://www.cdc.gov/coronavirus/2019-ncov/hcp/dental-settings.html

6. European Centre for Disease Prevention and Control. Guidance for wearing and removing personal protective equipment in healthcare settings for the care of patients with suspected or confirmed COVID-19. [acesso em 21 mai 2020]. Disponível em: https://www.ecdc.europa.eu/en/publicationsdata/guidance-wearing-and-removing-personal-protectiveequipment-healthcare-settings

7. ADA Interim Guidance for Minimizing Risk of COVID-19 Transmission. [acesso em 2 mai 2020]. Disponível em: https://www.ada.org/ /media/CPS/
Files/COVID/ADA_COVID_Int_Guidance_Treat_Pts.pdfCDC. 8. Coronavirus Disease 2019 (COVID-19). CDNA National Guidelines for Public Health Units. [acesso em 5 mai 2020]. Disponível em: https://www1.health.gov.au/internet/main/publishing.nsf/ Content/7A8654A8CB144F5FCA2584F8001F91E2/\$File/COVID-19SoNG-v2.10.pdf

9. National Patient Safety Agency England and National Patient Safety Agency Improvement - COVID-19 guidance and standard operating procedure Urgent dental care systems in the context of coronavirus- Delay phase. [acesso em 21 mai 2020]. Disponível em: https://www.england.nhs. uk/coronavirus/wp-content/uploads/sites/52/2020/04/C0282-covid-19urgent-dental-care-sop.pdf

10. World Health Organization (WHO). Rational use of personal protective equipment for coronavirus disease 2019 (COVID-19). [acesso em 21 mai 2020]. Disponível em: https://www.who.int/publications-detail/rational-use-of -personal-protective-equipment-for-coronavirus-disease-(covid-19)-and considerations-during-severe-shortages.

11. Public Health Agency of Canada (PHAC). Coronavirus disease (COVID-19): For health professionals. [acesso em 21 mai 2020]. Disponível em: https://www.canada.ca/en/public-health/services/diseases/2019novel-coronavirus-infection/health-professionals/infection-preventioncontrol-covid-19-second-interim-guidance.html\#a8.7.

12. Asadi S, Bouvier N, Wexler A S, Ristenpart W D. The coronavirus pandemic and aerosols: Does COVID-19 transmit via expiratory particles? Aerosol Sci Technol. 2020;54(6):635-638.

13. Ningthoujam R, COVID 19 can spread through breathing, talking, study estimates, Current Medicine Research and Practice. In Press 2020. 


\section{Mini Curriculum and Author's Contribution}

1. Vanessa Paiva Reis - DDS. Contribution: Literature review, preparation, writing, and review of the manuscript. ORCID: 0000-0001-7682-547X

Submitted: 06/08/2020 / Accepted for publication: 06/10/2020

Corresponding author:

Vanessa de Paiva Reis

E-mail: vanepreis1@gmail.com 